\title{
Wie möchten Sie sterben?
}

\section{Hans Stalder}

Prof. Dr. med., Mitglied der Redaktion

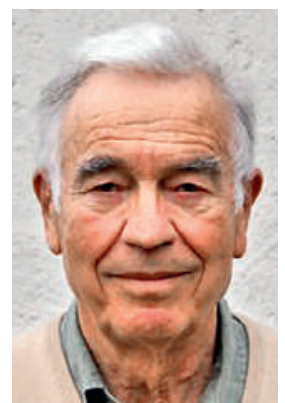

In ihrem Zu guter Letzt vom 25. November 2015 mit dem Titel Lieber jung, reich und gesund ... [1] teilt uns Anna Sax ihre Lebenserwartung mit, die sie mithilfe eines im Internet leicht zu findenden Fragebogens errechnet hat: Anna dürfte 98 Jahre alt werden! Ich habe dasselbe gemacht. Was für eine Enttäuschung: Ich komme nur auf 94 Jahre ... Mit einer kleinen Schummelei - ich habe mein Geschlecht auf dem Fragebogen geändert - erreiche ich fast dasselbe Alter wie Anna. Hätte sie ihren Beitrag eher mit Lieber jung, reich, gesund und weiblich betiteln sollen? Vielleicht nicht, denn auch wenn die Lebenserwartung der Frauen höher ist als die der Männer, ist sie mit mehr Erkrankungen assoziiert oder mindestens mit häufigeren Arztkonsultationen. Zudem fühlt sich Anna Sax beim Gedanken, dass sie nochmals 40 Jahre auf dieser Welt zu leben hat, etwas verunsichert [1].

Kardiale und Tumor-Ätiologien haben über das letzte Jahrzehnt hinweg stark abgenommen, während sich die Demenz fast verdreifacht hat.

Wenn man sein Umfeld fragt: «Wie möchten Sie sterben?», lautet die Antwort meist entweder unmittelbar oder nach kurzem Innehalten, aber mit Würde: auf keinen Fall in einem Zustand von Demenz oder mit Verlust der Autonomie. Gemäss Bundesamt für Statistik [2] verändern sich die Todesursachen und folglich auch die Lebensumstände unserer letzten Lebensjahre: kardiale und Tumor-Ätiologien haben über das letzte Jahrzehnt hinweg stark abgenommen, während sich die Demenz fast verdreifacht hat. Und nichts weist darauf hin, dass dieser Trend aufhört, denn zurzeit unternehmen wir alles, damit es so weitergeht. Unsere Gesundheitspolitik fördert energisch das Screening, und die Altersgrenzen für diesen oder jenen Eingriff werden ständig weiter nach oben verschoben [3]. Auch wenn ein Screening das Überleben oft nicht verlängert [4], senkt es doch tendenziell die Häufigkeit von Krebs und Infarkten, ohne dass wir wissen, welche Qualität die so gewonnene Lebenszeit haben und wie das Sterben ohne diese Krankheiten sein wird. Logischerweise müsste die Prävalenz der Demenz zunehmen trotz der Tatsache, dass ihre Inzidenz abzunehmen scheint. Es bringt auch nichts, hier den Begriff der quality adjusted life years (QALYs) ins Feld zu führen, denn De- menzkranke haben kaum die Möglichkeit, ihre Lebensqualität zu beurteilen, oder wenn sie dies noch können, uns das mittels eines Formulars mitzuteilen. Ein weiteres Beispiel (für all jene, die rasch sterben möchten): An öffentlichen Plätzen werden überall Defibrillatoren aufgestellt, um Leben $\mathrm{zu}$ retten. Auch wenn die durchschnittliche Überlebensrate nach einer Hospitalisation nur bei etwa 10\% liegt [5] - in gewissen Statistiken mit einer ausgeklügelten Methodik erzielt man leicht bessere Ergebnisse -, ist die Wahrscheinlichkeit gross, das jene, die das Spital lebend verlassen, an neurologischen Problemen bzw. Demenz leiden. Die Lebensqualität der Überlebenden ist im Übrigen leider kaum erforscht. Und ist es nicht etwas abwegig, wenn gewisse Geriater das beklagenswerte Leben von Demenzkranken mit Medikamenten noch verlängern wollen?

Obwohl der Tod keine medizinische Angelegenheit sein sollte [6], erfolgt er paradoxerweise häufig im Spital, und die letzten Wochen des Lebens verursachen sehr hohe Kosten für unser Gesundheitswesen. Es ist, als ob der Tod nicht etwas Normales wäre, sondern eine äusserst schwere Krankheit, die behandelt werden muss. Aber eigentlich erfordert er vor allem Pflege. Und diese Pflege sollte wohl eher durch Pflegende als durch Ärzte geleistet werden, denn letztere könnten unsere Autonomie infrage stellen, wenn wir unsere Tage beenden möchten [7], denn Fürsorge ist nicht immer dasselbe wie Wohltätigkeit.

Vergessen wir nicht, wenn es um Prävention im Alter oder um Pflege während der letzten Lebenstage geht, unseren älteren Patienten die Frage zu stellen, auch wenn dies schwierig ist: «Wie möchten Sie sterben?»

Literatur

1 Sax A. Lieber jung, reich und gesund ... Schweiz Ärztezeitung 2015;96:1790

2 http://www.statistik.admin.ch/

3 Münzer T. Gesundheitsprävention im Alter - Wer gut beraten wird, lebt länger! Swiss med Forum 2016;16:7-8.

4 Saquib N, Saquib J, Ioannidis JP. Does screening for disease save lives in asymptomatic adults? Systematic review of metaanalyses and randomized trials. Int J Epidemiol. 2015;44:264-77.

5 Savastano S, Klersy C, Raimondi M, Langord K, Vanni V, Rordorf $\mathrm{R}$, Vicentini A, Petracci B, Landolina M, Visconti LO. Positive trend in survival to hospital discharge after out-of-hospital cardiac arrest: a quantitative review of the literature. N Engl J Med 2014;15:609-15.

6. Berner D. A propos de la récente polémique sur l'assistance au suicide. BMS 2015;96:1930.

7 Borasio GD. Suizidhilfe aus ärztlicher Sicht - die vernachlässigte Fürsorge. BMS 2015;96:889-91. 